Research Article

\title{
Study on the Mechanism of Salvia miltiorrhiza in the Treatment of Traumatic Bone Defects
}

\author{
Qian Tan, Yaoxi Liu, Ting Lei, Weihua Ye, Xin Hu, Haibo Mei $\mathbb{D}^{\text {, }}$, and Ge Yang \\ Pediatric Orthopedic Lab, Department of Orthopedic Surgery, The Hunan Children's Hospital, Changsha 410007, Hunan, China \\ Correspondence should be addressed to Haibo Mei; meihaiboprof@outlook.com and Ge Yang; yangge@csu.edu.cn
}

Received 8 September 2021; Accepted 18 September 2021; Published 1 October 2021

Academic Editor: Weiguo Li

Copyright (c) 2021 Qian Tan et al. This is an open access article distributed under the Creative Commons Attribution License, which permits unrestricted use, distribution, and reproduction in any medium, provided the original work is properly cited.

\begin{abstract}
Traumatic bone defect is one of the major orthopedic diseases in clinics, and its incidence is increasing year by year. And repairing traumatic bone defects is a very difficult problem in clinics at present. The surface of medical titanium-based alloy has good biological properties, and its implant has a certain role in promoting bone in bone tissue. However, titanium-based materials are biologically inert and have no biological activity. As a traditional Chinese medicine, Salvia miltiorrhiza has the efficacy of treating bone diseases and promoting bone healing. The curative effect can be better exerted by loading the traditional Chinese medicine active compound Salvia miltiorrhiza on the surface of the titanium implant in a certain way. At present, due to the complex chemical composition of Salvia miltiorrhiza, the mechanism of its use for the treatment of traumatic bone defects is still unclear. Therefore, in this study, we mainly discussed the potential target and mechanism of Salvia miltiorrhiza in the treatment of traumatic bone defects through network pharmacology, which may provide a scientific basis for the treatment of traumatic bone defects with Salvia miltiorrhiza loaded on the surface of medical titanium-based alloy. We screened out effective compounds and targets of Salvia miltiorrhiza and targets related to traumatic bone defects with the help of relevant databases. The targets of Salvia miltiorrhiza for traumatic bone defects were analyzed by STRING and GeneCards databases, and the results were visualized by constructing a compound-target network, protein-protein interaction network, and compound-target-disease network with Cytoscape 3.7.1 analysis software. Finally, the selected core targets carried out GO and KEGG enrichment. The results showed that 60 main active components were screened from Salvia miltiorrhiza Bunge, which could act on 149 targets. There were 33 active components and 70 targets related to traumatic bone defects, respectively. The core targets of Salvia miltiorrhiza in the treatment of traumatic bone defects were MAPK1, MAPK10, MAPK14, TGFB1, and TNF. The results of enrichment analysis showed that Salvia miltiorrhiza might treat traumatic bone defects through an osteogenic differentiation pathway.
\end{abstract}

\section{Introduction}

Bone is an organ that maintains the normal posture and movement of the human body and has the function of protecting internal organs [1]. Traumatic bone defect is one of the most common, most frequent, and serious diseases in orthopedics and traumatology and even poses a certain threat to patients' lives in severe cases [2]. In recent years, with the continuous development of transportation and construction industry, as well as the deepening of the aging of the population, the incidence of traumatic bone defects is increasing, which has seriously affected the quality of life [3]. The repair and treatment of traumatic bone defects is a very difficult problem in clinical practice at present, and the treatment of bone defects is mainly through surgery and drug treatment. With the application of orthopedic implants in tissue engineering and the emergence of drugs on the surface of many implants, the combination of traditional Chinese medicine for bone repair and modern biomaterials for bone repair has become a promising treatment for bone defects [4]. Bone defects must be filled and implanted with appropriate substances to accelerate bone repair [5]. Autograft is the preferred filler material due to its biocompatibility and bone induction potential, but its application is limited by the limited number of autologous bones and the surgical trauma it can cause at the donor site [6]. Therefore, at present, we mainly use synthetic bone materials instead of autograft for filling, so as to realize the complete integration 
of nonliving implants and living bones [7]. Among them, medical titanium-based alloy is a new generation of medical metal materials developed after medical stainless steel and medical Co-Cr-Mo alloy, which has more excellent biocompatibility and other properties and is more suitable as an implant for the treatment of bone defects [8]. Drug treatment is mainly the use of traditional Chinese medicine internal and external application, the combination of western medicine, etc. The external traditional Chinese medicine can directly reach the focus and has stronger targeting, which can improve the drug concentration in the bone microenvironment [9]. At the same time, the traditional Chinese medicine can avoid liver first-off effect generated after the oral administration of drugs and has the advantages of no gastrointestinal tract stimulation, small toxic and side effects, good patient compliance, and the like so that the traditional Chinese medicine has better clinical effect and more advantages in treating traumatic bone defects [10]. The implant is a biologically inert material with no biological activity and can exert its curative effect by loading externally applied Chinese herbs with bone-promoting effects on the implant. Salvia miltiorrhiza is a traditional Chinese medicine and has certain curative effects in treating bone diseases and promoting bone healing. Salvia miltiorrhiza loaded on the surface of the titanium implant in a certain way can better exert the curative effect. However, the components of Salvia miltiorrhiza Bunge are complex so that the mechanism of Salvia miltiorrhiza in the treatment of traumatic bone defects is still unclear, which hinders the application and promotion of Salvia miltiorrhiza in the treatment of bone defects to a certain extent. On the basis of the biological network, Chinese medicine network pharmacology analyzes the relationship of the compound, target, and disease in the network by means of database retrieval, high-throughput omics data analysis, and computer simulation to reflect and describe the interaction [11]. In this work, we explored the potential mechanism of Salvia miltiorrhiza in the treatment of traumatic bone defects through network pharmacology, which played a positive role in promoting the clinical application of Salvia miltiorrhiza in the treatment of bone defects and the development of related drugs.

\section{Methods}

2.1. Screening of Effective Components and Targets of Salvia miltiorrhiza. We used the Chinese Medicine System Pharmacology Database (TCMSP) to screen. We entered Salvia miltiorrhiza under the column "Herb name" to perform search and set the screening conditions as oral bioavailability $\mathrm{OB} \geq 30 \%$, and drug-likeness $\mathrm{DL} \geq 0.18$. And we obtained the qualified active components of Salvia miltiorrhiza [12]. Target proteins related to effective active ingredients of Salvia miltiorrhiza were searched separately under the column of "Related Targets." With the help of the UniProt database (https://www.uniprot.org/), we standardized the human gene name corresponding to the target protein and finally obtained the target of Salvia miltiorrhiza.
2.2. Screening of Related Targets for Traumatic Bone Defects and Potential Core Targets for Treatment. We searched the disease-related targets in the GeneCards database and OMIM database with Traumatic bone defect and Traumatic fracture as key words, respectively. Then, we merged and removed the duplicate targets to get the related targets of the traumatic bone defect. The online Venny 2.1 Venn diagram was used to intersect the disease-related targets and drug targets. After that, we obtained the intersection targets of Salvia miltiorrhiza and traumatic bone defect, which were the potential therapeutic targets of Salvia miltiorrhiza in treating traumatic bone defects.

\subsection{Construction of the Compound-Target Network and} Protein-Protein Interaction Network. We constructed the Chinese medicine regulatory network of "drug-active ingredient-disease-target" using Cytoscape V3.8.2 software for visualization [13]. Finally, we obtained the most effective active ingredients according to the degree analysis. In order to clarify the role of target protein at the level of the biological network system, we introduced the target of Salvia miltiorrhiza in treating traumatic bone defects obtained in Section 2.2 into STRING (https://string-db.org/cgi/input.pl) to construct the protein-protein interaction network. The setting conditions were as follows: we chose Homo sapiens for species, deleted the free target, and set the minimum confidence level to 0.4 . We downloaded the corresponding TSV file and imported it into Cytoscape to generate the PPI network and used CytoNCA plugin to analyze the PPI network [14].

2.4. Enrichment Analysis. We used DAVID databases (https://David.ncifcrf.gov/home.jsp) to perform GO (gene ontology) functional enrichment analysis of core targets [15]; GO functional analysis describes the function of genes, which is divided into molecular biological function (MF), biological process (BP), and cellular components (CC). The KEGG database was used for pathway enrichment analysis of core targets to comprehensively predict the regulatory pathways of Salvia miltiorrhiza in the treatment of traumatic bone defects [16].

\section{Results}

3.1. Composition and Target of Salvia miltiorrhiza and Compound-Target Network. We used the Chinese Medicine System Pharmacology Database (TCMSP) to search the components of Salvia miltiorrhiza with setting the screening conditions as oral bioavailability $\mathrm{OB} \geq 30 \%$ and drug-likeness $\mathrm{DL} \geq 0.18$. We obtained 65 kinds of active ingredients through screening and then searched the corresponding target proteins of these 65 components. Five of these 65 components have no corresponding targets. Finally, we integrated targets and removed the duplicate targets to get a total of 149 targets which were the target points of Salvia miltiorrhiza. With Cytoscape V3.8.2 software, we built a compound-target network for visualization. As shown in Figure 1, the square node in the middle represents the target, and the darker the square node is, the more compounds act on it. The round nodes represent the 


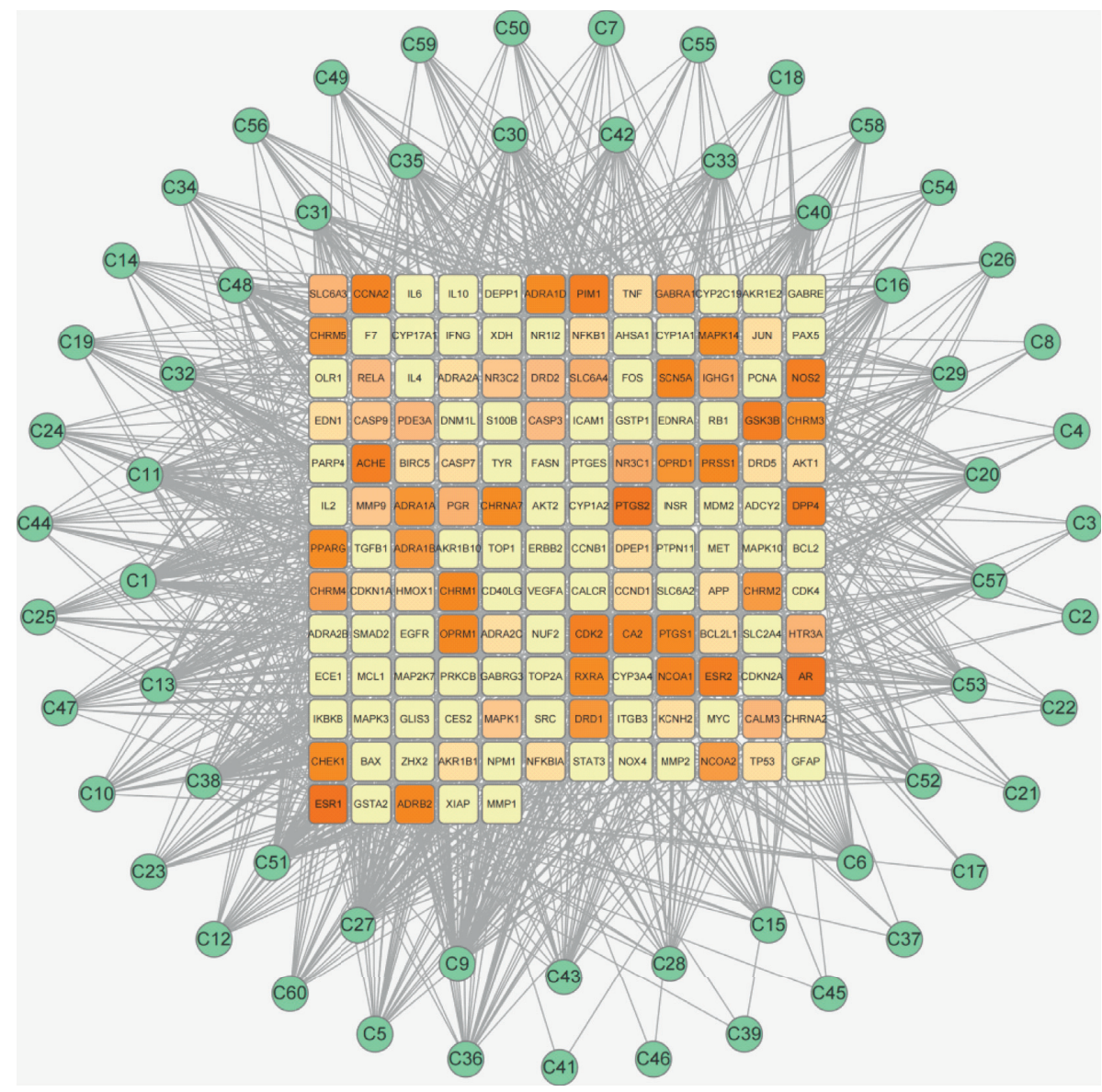

Figure 1: Compound-target network.

active components in Radix Salviae Miltiorrhizae, and the lines represent the interaction relationship between the active components and the targets. In the network, the components with more targets, which had darker color in the network diagram, may play an important role in the pharmacological function of Salvia miltiorrhiza.

\subsection{The Core Target of Salvia miltiorrhiza in the Treatment of} Traumatic Bone Defects. We searched the disease-related targets in the GeneCards database and OMIM database with Traumatic bone defect and Traumatic fracture as key words, respectively, and obtained 1700 targets in the GeneCards database and 608 targets in the OMIM database. We finally obtained 1482 targets related to traumatic bone defects after merging and removing the duplicate targets. As shown in Figure 2, we used Venny 2.1 to intersect these 1482 diseaserelated targets with the above 149 drug targets to get 70 common targets, which were potential therapeutic targets of Salvia miltiorrhiza for traumatic bone defects.

3.3. Protein-Protein Interaction Network. We introduced the above 70 common target genes into STRING and chose Homo sapiens for species, deleted the free target, and set the minimum confidence level to 0.4 . Through the information of the database, we obtained the interaction relationship between the protein and constructed PPI network, as shown in Figure 3. There were 70 nodes and 895 lines in the network graph, and the average nodal degree was 25.6. The nodes represented target proteins, and each line represented the interaction between target proteins. We processed the downloaded TSV file with CytoNCA plugin and finally obtained 14 core target proteins such as MAPK1, MAPK10, MAPK14, TGFB1, TNF, JUN, ITGB3, CALCR, FOS, AKT1, PPARG, IFNG, RELA, and NFNB1 (Figure 4). We associated these 14 core targets with the active components of Salvia miltiorrhiza and found that 33 active components of Salvia miltiorrhiza involve these 14 targets. As shown in Figure 5, rectangular nodes represented key target genes, circular nodes represented active ingredients in Salvia miltiorrhiza, and lines represented the interaction relationship between active ingredients and targets. The more lines corresponding to the target meant that more active ingredients acted on the target. The more lines corresponding to the active ingredient represented that there were more targets for the action of this active ingredient. Therefore, the more active ingredients and targets corresponding to the lines may be the key active ingredients and targets of Salvia miltiorrhiza in the treatment of traumatic bone defects. Among them, the more important active components and targets were luteolin, $\alpha$-amyrin, cryptotanshinone, tanshinone IIA, PPARG, and MAPK14, respectively. 


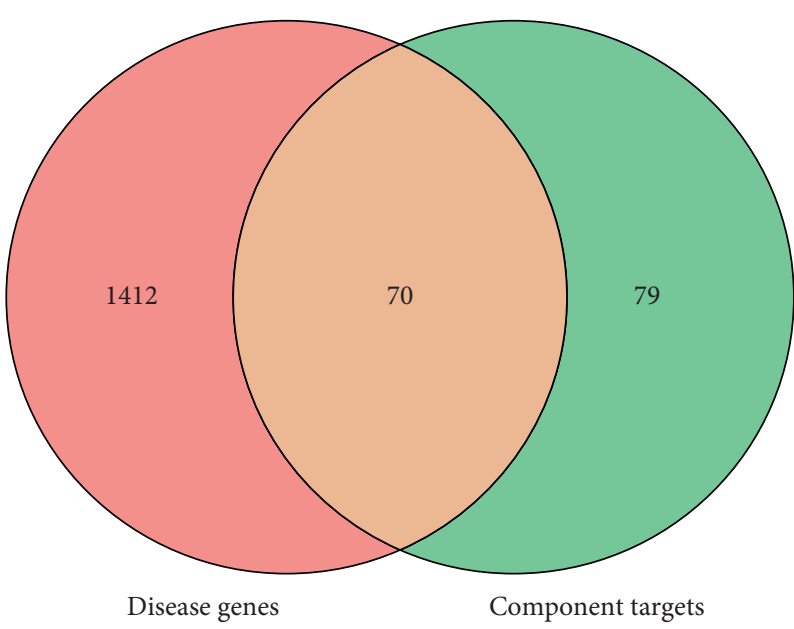

FIGURE 2: Potential therapeutic targets of Salvia miltiorrhiza for traumatic bone defects.



FIgURE 3: Network of interactions between proteins.

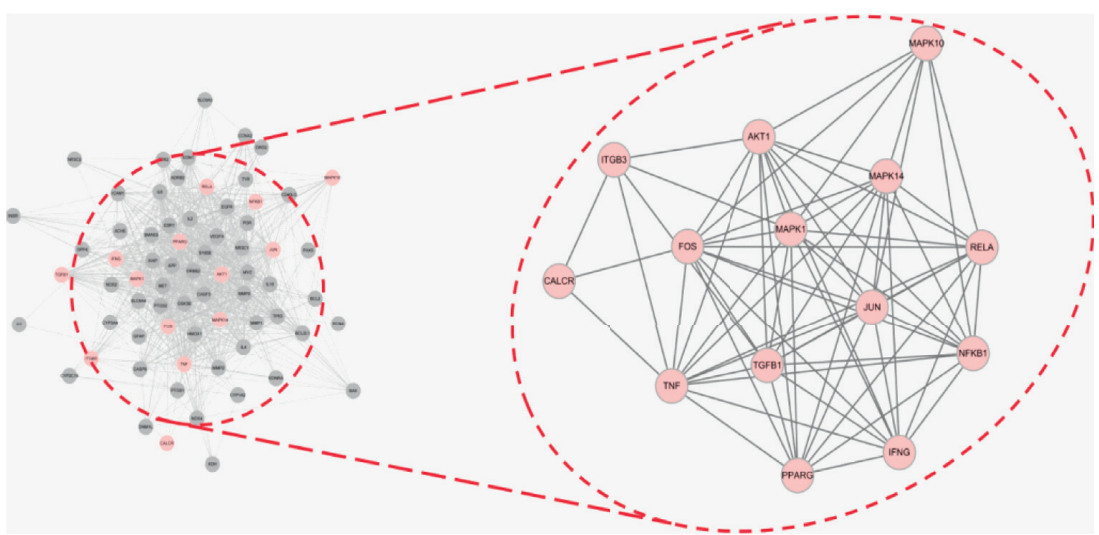

FIGURE 4: The key core targets of Salvia miltiorrhiza in the treatment of traumatic bone defects.

3.4. Enrichment Analysis. GO functional enrichment analysis consists of three parts: biological process, cellular component, and molecular function. We performed GO functional enrichment analysis on the common targets of the targets of Salvia miltiorrhiza and disease-related targets, as shown in Figure 6, with the vertical axis representing the 


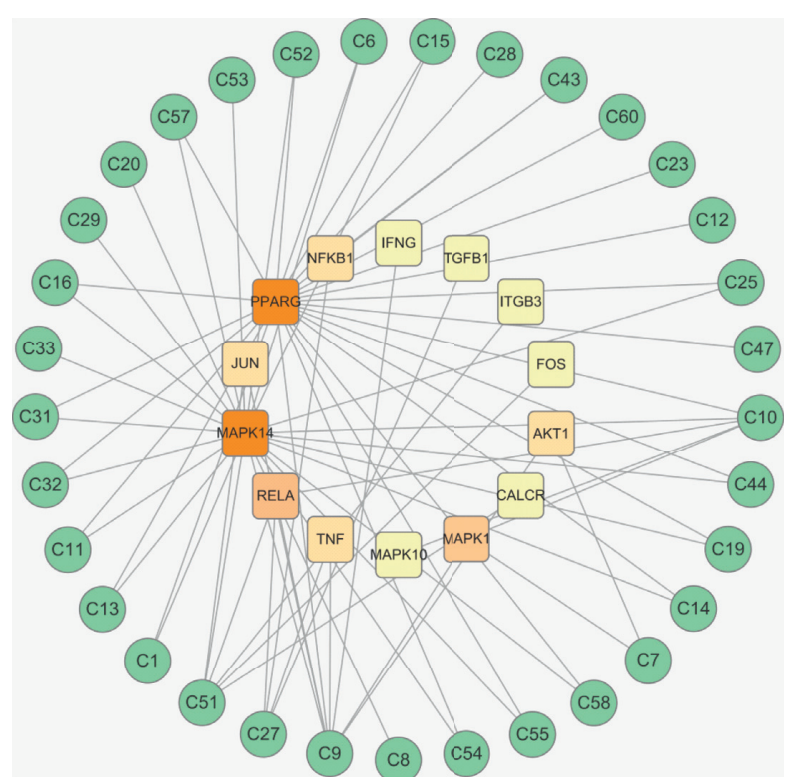

Figure 5: Active components corresponding to key core targets.

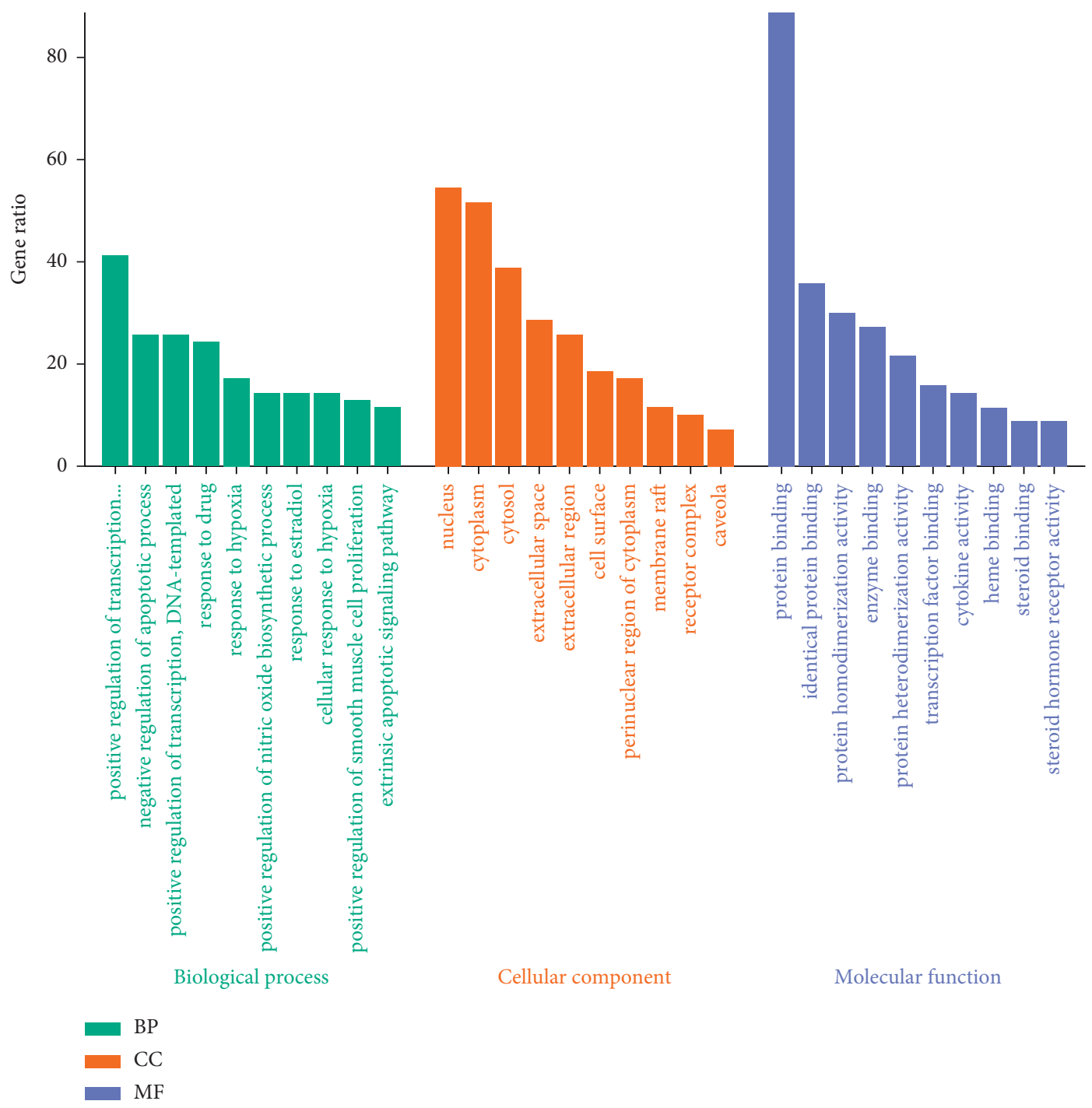

FIgURE 6: GO functional enrichment analysis of 149 targets. 


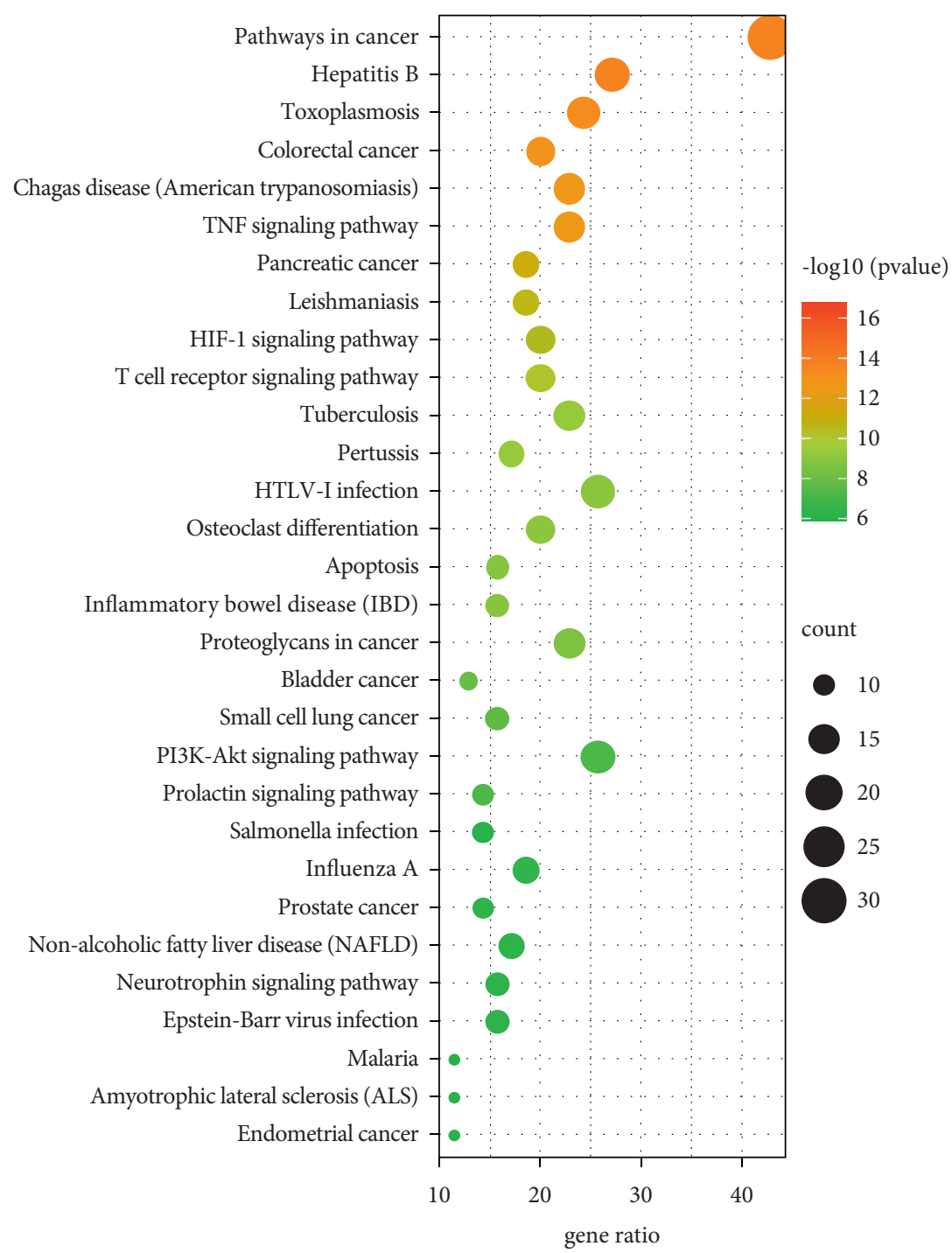

FIgURE 7: The KEGG pathway enrichment analysis of 149 targets.

name of the pathway and the horizontal axis representing the proportion of enriched genes. The results showed that biological processes involved in GO functional enrichment analysis were concentrated in the positive regulation of transcription from the RNA polymerase II promoter, response to the drug, positive regulation of the nitric oxide biosynthetic process, positive regulation of smooth muscle cell proliferation, extrinsic apoptotic signaling pathway in the absence of the ligand, positive regulation of transcription, DNA-templated, etc. The process of cellular components was mainly related to the extracellular space, cell surface, receptor complex, membrane raft, perinuclear region of the cytoplasm, nucleus, extracellular region, cytoplasm, and so on. And the process of molecular functions was mainly related to enzyme binding, identical protein binding, protein homodimerization activity, protein binding, protein heterodimerization activity, cytokine activity, steroid binding, transcription factor binding, heme binding, etc. 149 common target genes were analyzed by KEGG pathway enrichment analysis, and 111 pathways were obtained. Set the bar chart showing the first 30 paths with the smallest $p$ value, as shown in Figure 7. And in Figure 7, the abscissa represented the number of enrichment analyses, and the ordinate represented the $p$ value. The smaller the $p$ value, redder the color. The results of KEGG pathway enrichment analysis were mainly involved in osteoclast differentiation (hsa04380), apoptosis (hsa04210), PI3K-Akt signaling pathway (hsa04151), T-cell receptor signaling pathway (hsa04660), HIF-1 signaling pathway (hsa04066), TNF signaling pathway (hsa04668), amyotrophic lateral sclerosis (hsa05014), neurotrophin signaling pathway (hsa04722), and so on. Among them, osteoclast differentiation pathway (Figure 8) was closely related to the bone repair.

\section{Discussion}

Every year, more than 2 million people in the world suffer from bone defects with an economic burden of 3 billion dollars, which seriously affects the quality of life of patients. 


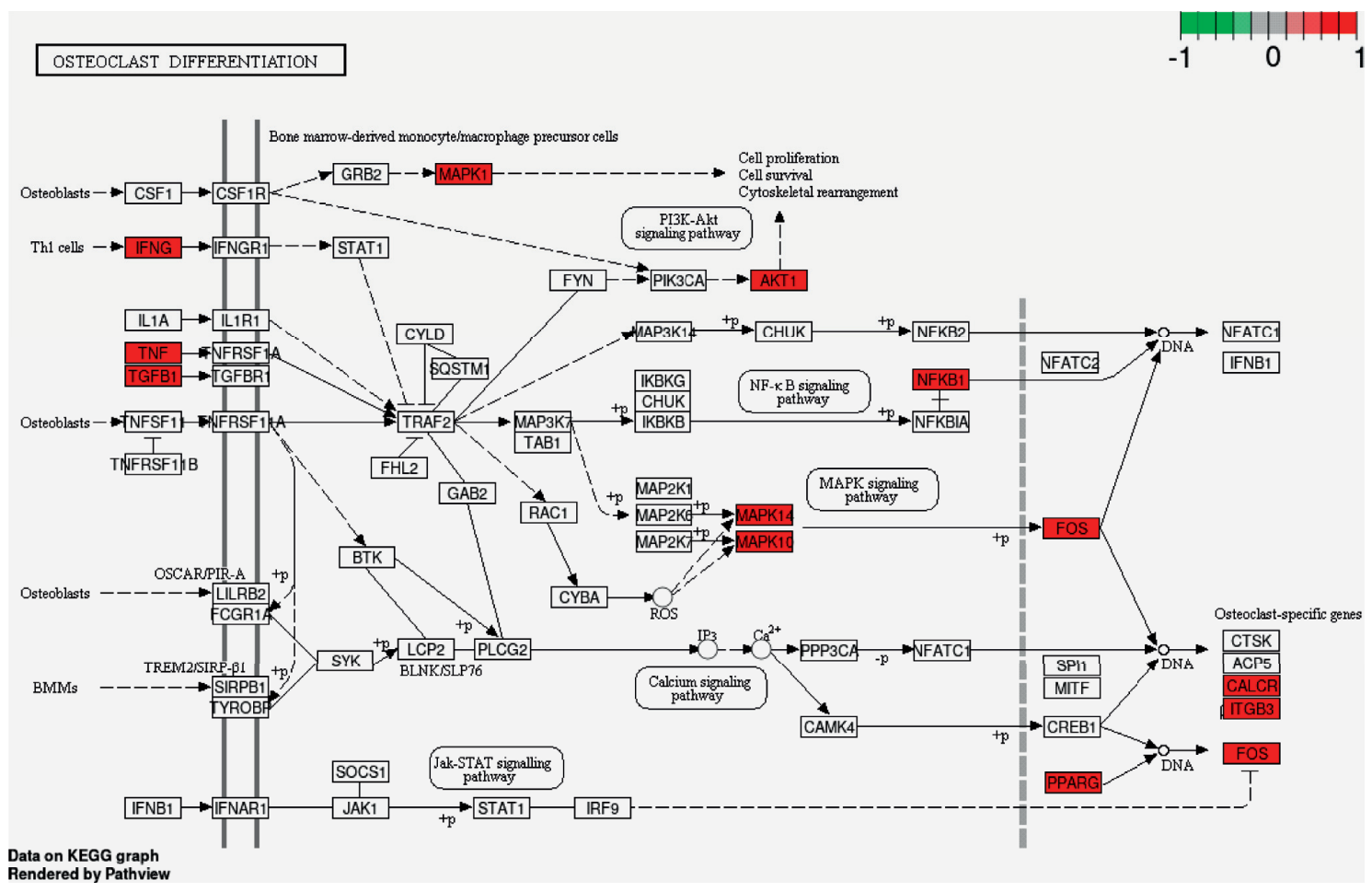

FIGURE 8: The diagram of the osteogenic differentiation pathway.

Bone transplantation is one of the most commonly used surgical operations. Autologous bone is the preferred filling material, but the number of autologous bones is limited [17]. At present, with the application of orthopedic implants in tissue engineering and the emergence of many drugs on the surface of implants, the combination of traditional Chinese medicine bone repair medicine and modern bone repair biomaterials has become a promising treatment method for bone defects. Salvia miltiorrhiza has the effect of repairing bone defects, but its mechanism is unclear because of its complex composition. This study preliminarily studied the possible mechanism of Salvia miltiorrhiza in treating traumatic bone defects by using the network pharmacology method. The results showed that Salvia miltiorrhiza had the characteristics of multicomponent and multitarget in treating traumatic bone defects, and one component of Salvia miltiorrhiza can act on multiple targets. According to the analysis of Cytoscape degree values, the ones with the highest component degree values were luteolin, $\alpha$-amyrin, cryptotanshinone, and tanshinone IIA, which may be the key components of Salvia miltiorrhiza in the treatment of traumatic bone defects. Bone repair depended on bone resorption and bone formation. Excessive osteoclast formation was not conducive to the repair of traumatic bone defects and led to a series of orthopedic diseases such as osteoporosis and rheumatoid arthritis. And our results were consistent with previous studies. Kim found that luteolin, which is a flavonoid compound, can inhibit the differentiation of bone marrow nuclear cells and Raw264.7 cells into osteoclasts and can reduce bone loss in osteoporosis by reducing the differentiation and function of osteoclasts [18]. $\alpha$-Amyrin is a pentacyclic triterpenoid. Related studies have shown that $\alpha$-amyrin can affect some pathways in skeletal muscle cells. Giacoman-Martínez et al. found that $\alpha$-amyrin can induce translocation of glucose transporter 4 (GLUT4) mediated by activated protein kinase (Ampk) and peroxisome proliferator-activated receptors (PPAR $\delta / \gamma)$ in $\mathrm{C} 2 \mathrm{C} 12$ myoblasts [19]. Cryptotanshinone belongs to a quinone compound. Studies showed that cryptotanshinone can alleviate osteoarthritis in mice. And its mechanism may be that cryptotanshinone can inhibit the production of PGE2 and NO induced by IL- $1 \beta$, inhibit the expression of ADAMTS-5, COX-2, MMP-3, MMP-13, and iNOS, and achieve it by inhibiting the activation of NF- $\kappa$ B and MAPK pathway [20]. The study showed that tanshinone IIA can inhibit the differentiation of osteoclasts and inhibit the differentiation of osteoclast precursor cells by inhibiting the expression of c-FOS and NFATC1 induced by the NF- $\kappa \mathrm{B}$ ligand receptor activator [21]. In this study, it was found that the key targets of Salvia miltiorrhiza in the treatment of traumatic bone defects were MAPK1, MAPK10, MAPK14, TGFB1, TNF, JUN, ITGB3, CALCR, FOS, AKT1, PPARG, IFNG, RELA, and NFNB1. FOS and JUN are members of the AP-1 transcription factor complex and are regulators of many cellular functions. JUN is associated with many inflammatory diseases [22]. FOS is a key regulator of bone cell differentiation, which can induce NFATC1 expression and inhibit osteoclast differentiation under the action of the active ingredient tanshinone IIA [21]. AKT1 is one of the key regulatory molecules of bone synthesis factor signaling and is highly expressed in bone cells [23]. Naohiro Kawamura et al. found that AKT1 can promote bone formation, and its mechanism may be through IGF-I and insulin-mediated osteoblast bone formation [24]. Tang et al. demonstrated 
that TGFB1 has the function of regulating bone resorption and promoting bone formation. And regulating TGFB1 may be an effective method for repairing bone defects. [25]. And PPARG can also regulate bone resorption and bone formation [26]. P38 mitogen-activated protein kinase (MAPK) signaling pathway plays an important role in regulating bone development and maintenance. MAPK14 belongs to the p38 mitogen-activated protein kinase family and is the most abundant member in bone cells in the p38 mitogen-activated protein kinase family [27]. We also found that osteoclast differentiation was an important signaling pathway through GO biological functions and KEGG pathway enrichment analysis, which corresponded to the above main components and targets. Therefore, the active components luteolin, $\alpha$-amyrin, cryptotanshinone, and tanshinone IIA in Radix Salviae Miltiorrhizae may play an important role in the treatment of traumatic bone defects, and its mechanism may be related to the regulation of MAPK1, MAPK10, MAPK14, TGFB1, TNF, JUN, ITGB3, CALCR, FOS, AKT1, PPARG, IFNG, RELA, and NFNB1 on the osteogenic differentiation pathway, so as to achieve the therapeutic effect. In this work, we preliminarily discussed the mechanism of Salvia miltiorrhiza in treating traumatic bone defects through network pharmacology, which laid a scientific foundation for the clinical application of Salvia miltiorrhiza. Math tools are very important in medical applications [28-31]. We think the work benefits the field.

\section{Conclusion}

In this study, we analyzed the mechanism of Radix Salviae Miltiorrhizae in the treatment of traumatic bone defects based on network pharmacology. We found a total of 65 active components in Radix Salviae Miltiorrhizae. Furthermore, through network analysis and screening, we obtained 33 main active components and 70 action targets, which were related to traumatic bone defects. The results showed that its mechanism of treating traumatic bone defects may be that luteolin, $\alpha$-amyrin, cryptotanshinone, and tanshinone IIA acted on MAPK1, MAPK10, MAPK14, TGFB1, TNF, JUN, ITGB3, CALCR, FOS, AKT1, PPARG, IFNG, RELA, and NFNB1 by the osteogenic differentiation pathway.

\section{Data Availability}

All the data supporting this work are included within the article.

\section{Conflicts of Interest}

The authors declare no conflicts of interest.

\section{Acknowledgments}

This work was supported by the Clinical Research Center for Limb Deformity of Children in Hunan Province (2019SK4006), the Major Science and Technology Projects for Collaborative Prevention and Control of Birth Defects in Hunan Province (2019SK1010), the Clinical Medical
Technology Innovation Guidance Project, Hunan Province (2020SK50517), and Natural Science Foundation of Hunan Province (2021JJ70081).

\section{References}

[1] K. Gulati, M. S. Aw, D. Findlay, and D. Losic, "Local drug delivery to the bone by drug-releasing implants: perspectives of nano-engineered titania nanotube arrays," Therapeutic Delivery, vol. 3, no. 7, pp. 857-873, 2012.

[2] P. Haentjens, J. Magaziner, C. S. Colón-Emeric et al., "Metaanalysis: excess mortality after hip fracture among older women and men," Annals of Internal Medicine, vol. 152, no. 6, pp. 380-390, 2010.

[3] S. Maher, A. Mazinani, M. R. Barati, and D. Losic, "Engineered titanium implants for localized drug delivery: recent advances and perspectives of titania nanotubes arrays," Expert Opinion on Drug Delivery, vol. 15, no. 10, pp. 1021-1037, 2018.

[4] L. Mohan, C. Anandan, and N. Rajendran, "Drug release characteristics of quercetin-loaded $\mathrm{TiO}_{2}$ nanotubes coated with chitosan," International Journal of Biological Macromolecules, vol. 93, pp. 1633-1638, 2016.

[5] M. Kikuchi, S. Itoh, S. Ichinose, K. Shinomiya, and J. Tanaka, "Self-organization mechanism in a bone-like hydroxyapatite/ collagen nanocomposite synthesized in vitro and its biological reaction in vivo," Biomaterials, vol. 22, no. 13, pp. 1705-1711, 2001.

[6] C. J. Damien and J. R. Parsons, "Bone graft and bone graft substitutes: a review of current technology and applications," Journal of Applied Biomaterials, vol. 2, no. 3, pp. 187-208, 1991.

[7] B. S. Liu, C. H. Yao, Y. S. Chen, and S. H. Hsu, "In vitro evaluation of degradation and cytotoxicity of a novel composite as a bone substitute," Journal of Biomedical Materials Research Part A, vol. 67A, no. 4, pp. 1163-1169, 2003.

[8] J. Li, Y. Ying, H. Xie et al., "Dual regulatory role of CCNA2 in modulating CDK6 and MET-mediated cell-cycle pathway and EMT progression is blocked by miR-381-3p in bladder cancer," The FASEB Journal, vol. 33, no. 1, pp. 1374-1388, 2019.

[9] S. Rahman, K. Gulati, M. Kogawa et al., "Drug diffusion, integration, and stability of nanoengineered drug-releasing implants in bone ex-vivo," Journal of Biomedical Materials Research Part A, vol. 104, no. 3, pp. 714-725, 2016.

[10] C.-Y. Lin, J.-S. Sun, S.-Y. Sheu, F.-H. Lin, Y.-J. Wang, and L.-T. Chen, "The effect of Chinese medicine on bone cell activities," American Journal of Chinese Medicine, vol. 30, no. 02n03, pp. 271-285, 2002.

[11] H. Yuan, Q. Ma, H. Cui et al., "How can synergism of traditional medicines benefit from network pharmacology?" Molecules, vol. 22, no. 7, p. 1135, 2017.

[12] J. Ru, P. Li, J. Wang et al., "TCMSP: a database of systems pharmacology for drug discovery from herbal medicines," Journal of Cheminformatics, vol. 6, no. 1, pp. 13-16, 2014.

[13] P. Shannon, A. Markiel, O. Ozier et al., "Cytoscape: a software environment for integrated models of biomolecular interaction networks," Genome Research, vol. 13, no. 11, pp. 2498-2504, 2003.

[14] Y. Tang, M. Li, J. Wang, Y. Pan, and F.-X. Wu, "CytoNCA: a cytoscape plugin for centrality analysis and evaluation of protein interaction networks," Biosystems, vol. 127, pp. 67-72, 2015.

[15] G. Dennis, B. T. Sherman, D. A. Hosack et al., "DAVID: database for annotation, visualization, and integrated discovery," Genome Biology, vol. 4, no. 5, pp. P3-P11, 2003. 
[16] M. Kanehisa and S. Goto, "KEGG: kyoto encyclopedia of genes and genomes," Nucleic Acids Research, vol. 28, no. 1, pp. 27-30, 2000.

[17] W. Sun, M. Li, L. Xie et al., "Exploring the mechanism of total flavonoids of drynariae rhizoma to improve large bone defects by network pharmacology and experimental assessment," Frontiers in Pharmacology, vol. 12, p. 1303, 2021.

[18] T.-H. Kim, J. W. Jung, B. G. Ha et al., "The effects of luteolin on osteoclast differentiation, function in vitro and ovariectomy-induced bone loss," The Journal of Nutritional Biochemistry, vol. 22, no. 1, pp. 8-15, 2011.

[19] A. Giacoman-Martínez, F. Javier Alarcón-Aguilar, A. Zamilpa et al., " $\alpha$-amyrin induce GLUT4 translocation mediated by AMPK and PPAR $\delta / \gamma$ in C2C12 myoblasts," Canadian Journal of Physiology and Pharmacology, vol. 99, no. 9, 2021.

[20] Z. Feng, W. Zheng, X. Li et al., "Cryptotanshinone protects against IL- $1 \beta$-induced inflammation in human osteoarthritis chondrocytes and ameliorates the progression of osteoarthritis in mice," International Immunopharmacology, vol. 50, pp. 161-167, 2017.

[21] H. B. Kwak, D. Yang, H. Ha et al., "Tanshinone IIA inhibits osteoclast differentiation through down-regulation of c-Fos and NFATc1," Experimental \& Molecular Medicine, vol. 38, no. 3, pp. 256-264, 2006.

[22] E. Wagner, "Bone development and inflammatory disease is regulated by AP-1 (Fos/Jun)," Annals of the Rheumatic Diseases, vol. 69, no. Suppl 1, pp. i86-i88, 2010.

[23] M. Almeida, L. Han, T. Bellido, S. C. Manolagas, and S. Kousteni, "Wnt proteins prevent apoptosis of both uncommitted osteoblast progenitors and differentiated osteoblasts by $\beta$-Catenin-dependent and -independent signaling cascades involving src/ERK and phosphatidylinositol 3-kinase/AKT," Journal of Biological Chemistry, vol. 280, no. 50, pp. 41342-41351, 2005.

[24] N. Kawamura, F. Kugimiya, Y. Oshima et al., "Akt1 in osteoblasts and osteoclasts controls bone remodeling," PLoS One, vol. 2, no. 10, Article ID e1058, 2007.

[25] Y. Tang, X. Wu, W. Lei et al., "TGF- $\beta 1$-induced migration of bone mesenchymal stem cells couples bone resorption with formation," Nature Medicine, vol. 15, no. 7, pp. 757-765, 2009.

[26] L. A. Stechschulte, P. J. Czernik, Z. C. Rotter et al., "PPARG post-translational modifications regulate bone formation and bone resorption," EBioMedicine, vol. 10, pp. 174-184, 2016.

[27] C. Thouverey and J. Caverzasio, "Focus on the p38 MAPK signaling pathway in bone development and maintenance," Bonekey Reports, vol. 4, 2015.

[28] M. Z. Yang, B. B. Zhang, J. C. Huang et al., "Network pharmacology reveals polyphyllin II as one hit of nano Chinese medicine monomers against nasopharyngeal carcinoma," Bioinorganic Chemistry and Applications, vol. 2021, Article ID 9959634, 10 pages, 2021.

[29] N. Xu, B. B. Zhang, X. N. Huang et al., "S100A8/A9 molecular complexes promote cancer migration and invasion via the $\mathrm{p} 38$ MAPK pathway in nasopharyngeal carcinoma," Bioinorganic Chemistry and Applications, vol. 2021, Article ID 9913794, 11 pages, 2021.

[30] X. He, L. Chen, H. Chen, Y. Feng, B. Zhu, and C. Yang, "Diagnostic accuracy of procalcitonin for bacterial infection in liver failure: a meta-analysis," Bioinorganic Chemistry and Applications, vol. 2021, Article ID 5801139, 8 pages, 2021.

[31] M. Liu, X. Lin, Q. Tan, and X. Han, "Evidence-based analysis of the emergency temporary cardiac pacing (electrical stimulation from metal wire electrode)," Bioinorganic Chemistry and Applications, vol. 2021, Article ID 5677598, 9 pages, 2021. 